\title{
Single trial ERP reading based on parallel factor analysis
} \author{
SABINE VAN HUFFEL, ${ }^{\mathrm{a}, \mathrm{b}}$ AND MAARTEN DE VOS $^{\mathrm{a}, \mathrm{k}}$ \\ ${ }^{a}$ Department of Electrical Engineering, ESAT-SCD, Katholieke Universiteit Leuven, Leuven, Belgium \\ ${ }^{b}$ IBBT-KU Leuven Future Health Department, Leuven, Belgium \\ ${ }^{c}$ Laboratory of Experimental Psychology, Katholieke Universiteit Leuven, Leuven, Belgium \\ ${ }^{\mathrm{d} B i o s c i e n c e s}$ and Technology Department, MOBILAB, Katholieke Hogeschool Kempen, Geel, Belgium \\ ${ }^{\mathrm{e}}$ Faculty of Psychology and Neuroscience, Maastricht University, Maastricht, The Netherlands \\ ${ }^{f}$ Department of Psychology, Tilburg University, Tilburg, The Netherlands \\ ${ }^{\mathrm{g}}$ Department of Psychology, Katholieke Universiteit Leuven, Leuven, Belgium \\ ${ }^{\text {h}}$ Flemish Government, Department of Welfare, Public Health and Family, Brussels, Belgium \\ ${ }^{i}$ Department of Pediatric Neurology, Katholieke Universiteit Leuven, Leuven, Belgium \\ 'Department of Radiology, Katholieke Universiteit Leuven, Leuven, Belgium \\ ${ }^{\mathrm{k}}$ Department of Psychology, Neuropsychology Lab, Oldenburg University, Oldenburg, Germany
}

KATRIEN VANDERPERREN, ${ }^{\mathrm{a}, \mathrm{b}}$ BOGDAN MIJOVIĆ, ${ }^{\mathrm{a}, \mathrm{b}}$ NIKOLAY NOVITSKIY, ${ }^{\mathrm{c}}$ BART VANRUMSTE, ${ }^{\mathrm{a}, \mathrm{d}}$ PETER STIERS, ${ }^{\mathrm{e}}$ BEA R. H. VAN DEN BERGH, ${ }^{\mathrm{f}, \mathrm{g}, \mathrm{h}}$ LIEVEN LAGAE, ${ }^{\mathrm{i}}$ STEFAN SUNAERT, ${ }^{\mathrm{j}}$ JOHAN WAGEMANS,

\begin{abstract}
The extraction of task-related single trial ERP features has recently gained much interest, in particular in simultaneous EEG-fMRI applications. In this study, a specific decomposition known as parallel factor analysis (PARAFAC) was used, in order to retrieve the task-related activity from the raw signals. Using visual detection task data, acquired in normal circumstances and simultaneously with fMRI, differences between distinct task-related conditions can be captured in the trial signatures of specific PARAFAC components when applied to ERP data arranged in Channels $\times$ Time $\times$ Trials arrays, but the signatures did not correlate with the fMRI data. Despite the need for parameter tuning and careful preprocessing, the approach is shown to be successful, especially when prior knowledge about the expected ERPs is incorporated.
\end{abstract}

Descriptors: Cognition, Normal volunteers, EEG/ERP, fMRI/PET/MRI

Use of the electroencephalogram (EEG) is widespread for both clinical and experimental neuroimaging purposes. One particular field for which EEG is applied extensively is the study of eventrelated potentials (ERPs). The most straightforward approach to study these ERPs is to average over a set of event trials, thereby emphasizing the time-locked brain activity and canceling out the other ongoing neural processes and noise. This approach, however, assumes that the brain is always reacting in exactly the same way to a certain stimulus. This might not be true since processes like attention and habituation do influence the brain's responses (Wesensten, Badia, \& Harsh, 1990). Therefore, it is interesting that recent improvements in analysis techniques allow going beyond studying global effects with average ERPs and investigate fluctuations in single trial characteristics (e.g., Makeig et al., 2002).

This research is supported by the Research Council KU Leuven: GOA MaNet and CoE EF/05/006; IUAP P6/04 (DYSCO, 2007-2011); the Flemish Government: G.0427.10N Integrated EEG-fMRI, IWTTBM080658-MRI and IBBT; and Neuromath (COST-BM0601). K. Vanderperren is supported by a $\mathrm{PhD}$ grant from the Agency for Innovation by Science and Technology (IWT), M. De Vos by an Alexander von Humboldt grant, and J. Wagemans by long-term structural funding from the Flemish Government (METH/08/02).

Address correspondence to: Katrien Vanderperren, Kasteelpark Arenberg 10-Box 2446, B-3001 Leuven, Belgium. E-mail: katrien.vanderperren@esat.kuleuven.be
Moreover, a more particular interest in the extraction of information from single trial ERP data has risen since researchers began to focus on the relationship between ERPs and functional magnetic resonance imaging (fMRI) data. Several studies have included single trial ERP data in combined EEG-fMRI analyses (e.g., Bénar et al., 2007; Debener et al., 2005; Debener, Ullsperger, Siegel, \& Engel, 2006; Eichele et al., 2005). A major part of these studies are based on so-called integration-by-prediction analyses, using specific characteristics of ERP components on a single trial level as regressors in the analysis of fMRI data. Nevertheless, so far there is no gold standard for the estimation and validation of single trial responses.

Several studies have employed different blind source separation (BSS) methods in order to isolate task-related activity from the typical EEG mixture of brain and nonbrain sources (e.g., De Vos et al., 2010; De Vos, De Lathauwer, \& Van Huffel, 2011; Joyce, Gorodnitsky, \& Kutas, 2004). Independent component analysis (ICA), for example, not only allows extracting single trial ERP information (Makeig, Jung, Bell, Ghahremani, \& Sejnowski, 1997; Makeig et al., 1999), but can also relate this to simultaneously measured fMRI activations (Debener et al., 2005, 2006; Eichele, Calhoun, \& Debener, 2009). Methods like ICA, however, can be applied in the spatial or in the temporal domain, but they come with the inherent drawback of being only two dimensional. 
A method that is able to disentangle brain activity but is not limited to two dimensions is parallel factor analysis (PARAFAC) (Harshman, 1970). This method decomposes three-dimensional (or higher) data in a set of distinct "atoms," which ideally represent distinct brain sources. PARAFAC has already shown its value for the analysis of EEG data, for example, for the identification of activity in specific frequency bands (Miwakeichi et al., 2004), the localization of the seizure onset zone in epileptic data (De Vos et al., 2007; De Vos, De Lathauwer, Vanrumste, Van Huffel, \& Van Paesschen, 2007) and the determination of the location of neonatal brain seizures (Deburchgraeve et al., 2009). It has also found its way into the study of ERP data (Achim \& Bouchard, 1997; Field \& Graupe, 1991; Möcks, 1988a, 1988b; Mørup, Hansen, Herrmann, Parnas, \& Arnfred, 2006; Wang, Begleiter, \& Porjesz, 2000). Although these studies proved PARAFAC to be a useful alternative for the above-mentioned two-dimensional methods, no single trial information was yet included as PARAFAC was only applied to ERP measures that were averaged across trials. The current study brings together the advantages of PARAFAC with the search for task-related information in single trial ERPs. For this reason, PARAFAC is used to extract sources from three-dimensional data arrays with dimensions Channels $\times$ Time $\times$ Trials. Compared to earlier studies (De Vos et al., 2007; Miwakeichi et al., 2004; Mørup et al., 2006), no wavelet transformation is applied prior to decomposing the data. As such, the PARAFAC decomposition will be based on characteristic spatiotemporal patterns rather than spectral behavior.

More specifically, the performance of PARAFAC in identifying distinct task-related conditions in single trial ERP data is investigated at different levels of increasing difficulty. To this end, the decomposition was validated on ERPs from EEG data both acquired in a control room and recorded simultaneously with fMRI data inside an MR scanner. Since the latter data are more severely contaminated with artifacts, this allows testing the robustness of the method. In addition, based on the superior extraction of task-related information with PARAFAC, the resulting components are used to investigate the relationship between ERPs and fMRI.

\section{Materials and Methods}

\section{Subjects}

Twenty-seven healthy subjects (11 female and 16 male, aged $18-44$, mean $=26.4, S D=5.2$ ) with no history of neurological or cardiological disorders participated in this study. Written informed consent was obtained in accordance with the local ethical committee guidelines. Twenty-six of the subjects performed the experiments inside an MR scanner with simultaneous fMRI acquisition. Nineteen subjects (18 overlapping) performed the experiments outside the MR scanner in the MR control room. The order of testing in these two different recording environments was randomized across subjects.

\section{Task Paradigm}

The employed stimulation paradigm was presented to the participants with Presentation software (Neurobehavioral Systems, Albany, CA). More specifically, a visual detection paradigm was used in which segments of circular black and white checkerboard stimuli were presented one at a time in randomized sequences to one of the four quadrants of the visual field (Di Russo, Martinez,
Sereno, Pitzalis, \& Hillyard, 2002). The segments were $6^{\circ}$ $(3.4 \times 3.3 \mathrm{~cm})$ in size, and their centers were positioned at $8^{\circ}$ $(5 \mathrm{~cm})$ distance from the fixation point. The spatial frequency of the stimuli was 0.5 cycles/degree. In addition, a large circular black and white checkerboard with about $5^{\circ}$ radius was presented as a central stimulus on the middle part of the screen. The stimuli were presented in four blocks of 100 stimuli (20 of each type) and 61 empty events. Subjects were asked to press a button upon detection of each of the stimuli. In each condition, the stimulus was presented for $150 \mathrm{~ms}$ with an interstimulus interval (ISI) varying between $900 \mathrm{~ms}$ and 2,400 ms. More detailed information about the set-up of this task can be found in an earlier study (Novitskiy et al., 2011). All subjects reported the task as being easy to perform and on average only 0.51 and 0.80 of the stimuli per block were missed for the simultaneous and the nonsimultaneous measurements, respectively.

This task was chosen for this work since it is known to evoke robust $\mathrm{C} 1, \mathrm{P} 1$, and $\mathrm{N} 1$ components, showing different properties depending on the position in the visual field where the stimuli are shown. In particular, the contralateral P1 is known to precede the ipsilateral P1, whereas for the stimuli shown in the upper versus lower visual fields, differences are found in the early $\mathrm{C} 1$ component (inverting polarity between upper and lower stimuli) and the amplitudes of the P1 and N1 components (Di Russo et al., 2002).

\section{Data Acquisition}

Both inside and outside the scanner, the EEG data were collected from 62 standard scalp sites using the MR-compatible BrainAmp MR+ system (BrainProducts, Munich, Germany) with a sampling rate of $5 \mathrm{kHz}$. Two additional electrodes were placed below the left eye and on the left scapula. All 64 channels were recorded with $\mathrm{FCz}$ as reference and $\mathrm{Iz}$ as ground.

For the acquisition of fMRI data simultaneously with the EEG, a Philips 3T Intera whole-body scanner (Royal Philips Electronics, Amsterdam, The Netherlands) was used. One hundred and sixty echo-planar images (EPI) composed of 28 slices of $3 \times 3 \times 4.5 \mathrm{~mm}$ voxel size and $4.8 \mathrm{~mm}$ slice thickness were recorded with ascending slice order with $1.95 \mathrm{~s}$ repetition time (TR) and $33 \mathrm{~ms}$ echo time (TE) during each experimental block. In addition, a full brain anatomical image was obtained with the magnetization prepared rapid gradient echo (MPRAGE) imaging sequence (230 coronal slices, $\mathrm{TE}=4.6 \mathrm{~ms}, \mathrm{TR}=9.7 \mathrm{~s}$ ). In addition, 5 out of the 26 "inside" subjects also performed the same paradigm in a Siemens 3T Allegra scanner (Siemens, Munich, Germany).

\section{Data Preprocessing}

The acquired EEG data were subjected to a number of standard preprocessing steps, common for EEG simultaneously measured with fMRI data. In particular, gradient artifacts were removed with the average template subtraction method (Allen, Josephs, \& Turner, 2000), as implemented in the Bergen EEG-fMRI EEGLAB plug-in (Moosmann et al., 2009), and ballistocardiogram (BCG) artifacts were reduced with a combination of the Optimal Basis Set (OBS) method (Niazy, Beckmann, Iannetti, Brady, \& Smith, 2005) and ICA (Vanderperren et al., 2010). More details on this preprocessing can be found in the article of Novitskiy et al. (2011). In addition, both in simultaneously and nonsimultaneously recorded data, eye artifacts were reduced with ICA (Joyce et al., 2004), and data were rereferenced to the average of TP9 and TP10 (the closest electrodes 
(a)
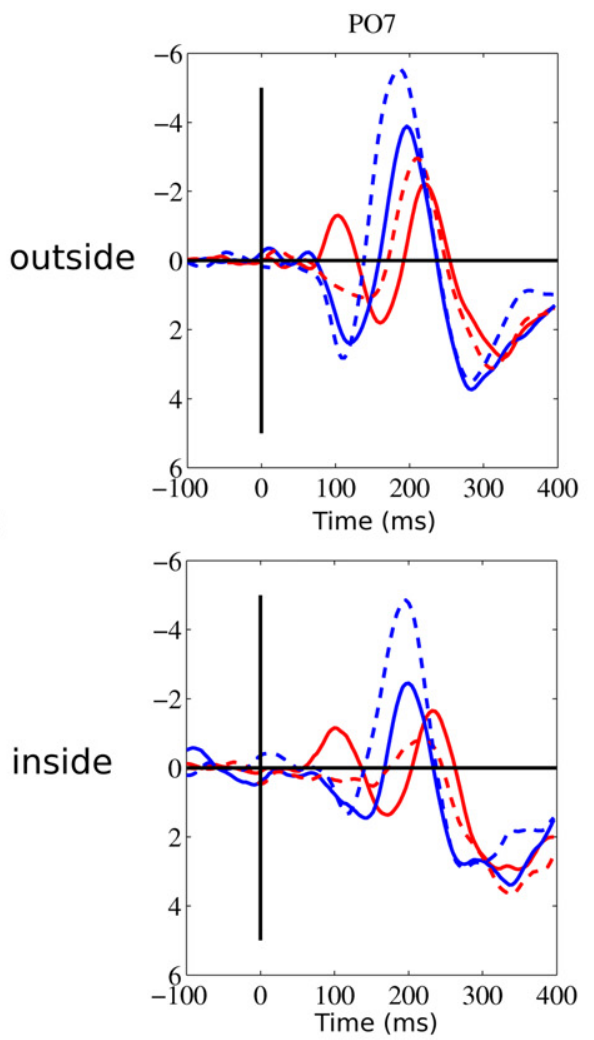
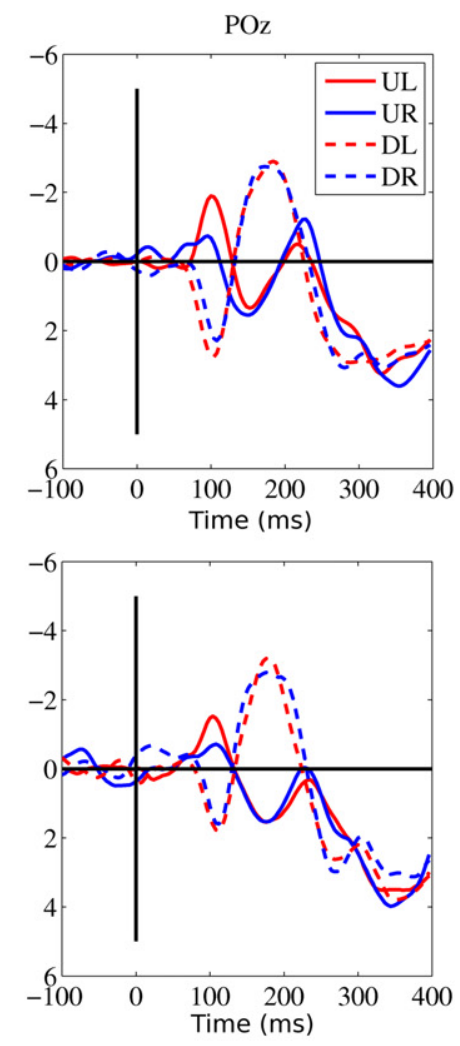
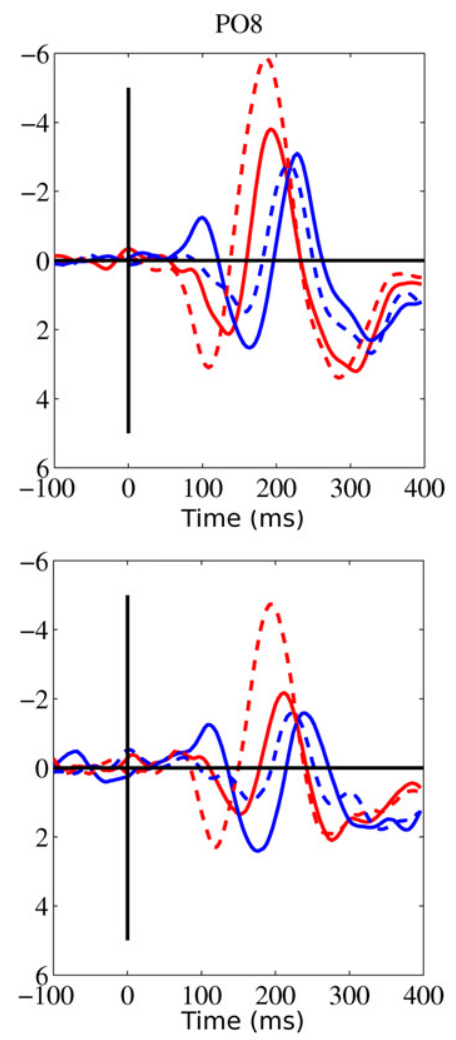

\section{P1 latency}

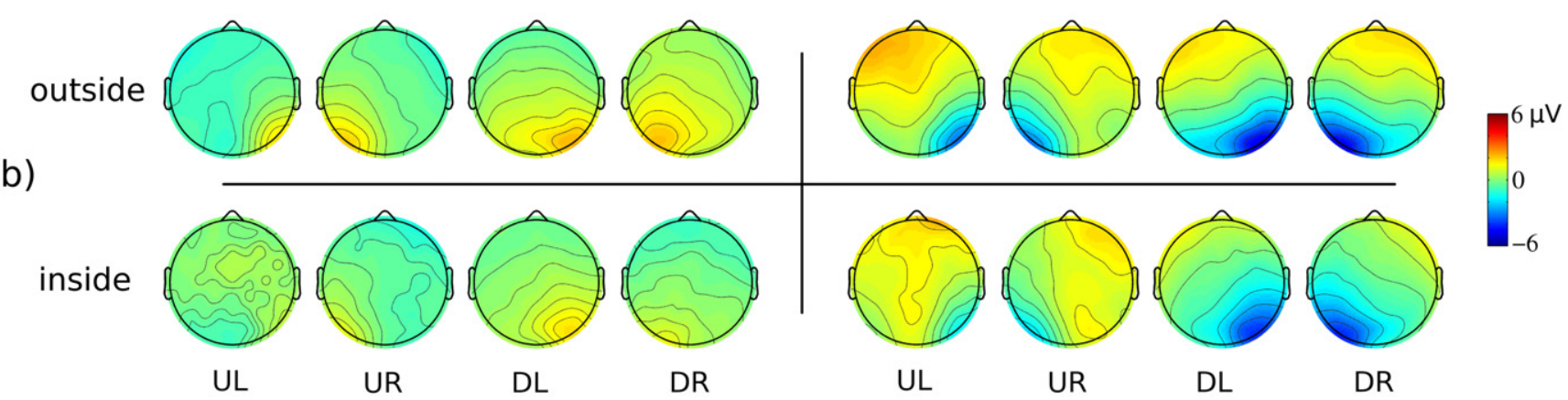

Figure 1. Grand mean ERPs for data acquired both simultaneously ("inside") and nonsimultaneously ("outside") with fMRI. a: ERP waveforms for the four peripheral stimuli (UL = upper left; UR = upper right; $\mathrm{DL}=$ down left; $\mathrm{DR}=$ down right) and for three different parietal-occipital electrodes; b: scalp topographies obtained from the ERPs around the latency of the P1 (left) and N1 (right), respectively.

to the mastoids in the present electrode set-up). To extract taskrelated ERPs, data were then segmented from $100 \mathrm{~ms}$ before until $400 \mathrm{~ms}$ after stimulus onset, baseline-corrected and thresholded on 50 or $100 \mu \mathrm{V}$ for measurements inside and outside the scanner, respectively. fMRI analysis was performed with the statistical parametric mapping software (SPM5, Wellcome Department of Cognitive Neurology, London, UK). The EPI time series were slice-time corrected, realigned, coregistered with anatomical images, normalized to a template, and smoothed with an 8-mm full width at half maximum (FWHM) Gaussian kernel.

\section{PARAFAC}

PARAFAC is a multidimensional decomposition technique that can decompose three-dimensional (or higher) signals into a series of distinct atoms or components (Smilde, Bro, \& Geladi, 2004). It can be seen as a higher-order generalization of a matrix singular value decomposition (Carroll \& Chang, 1970; Harshman, 1970). Every atom is characterized by a certain distribution or course in each of the modes. For the three-dimensional case, each element of the data array can be defined as follows:

$$
x_{d f t}=\sum_{k=1}^{N_{k}} a_{d k} \cdot b_{f k} \cdot c_{t k}+e_{d f t}
$$

with $N_{k}$ the number of atoms, $a_{d k}, b_{f k}$, and $c_{t k}$ the signatures of every atom in each of the modes, and $e_{d f t}$ the model error. In this study, PARAFAC was performed based on an alternating least squares algorithm (Smilde et al., 2004) with the N-way toolbox (Andersson \& Bro, 2000). 

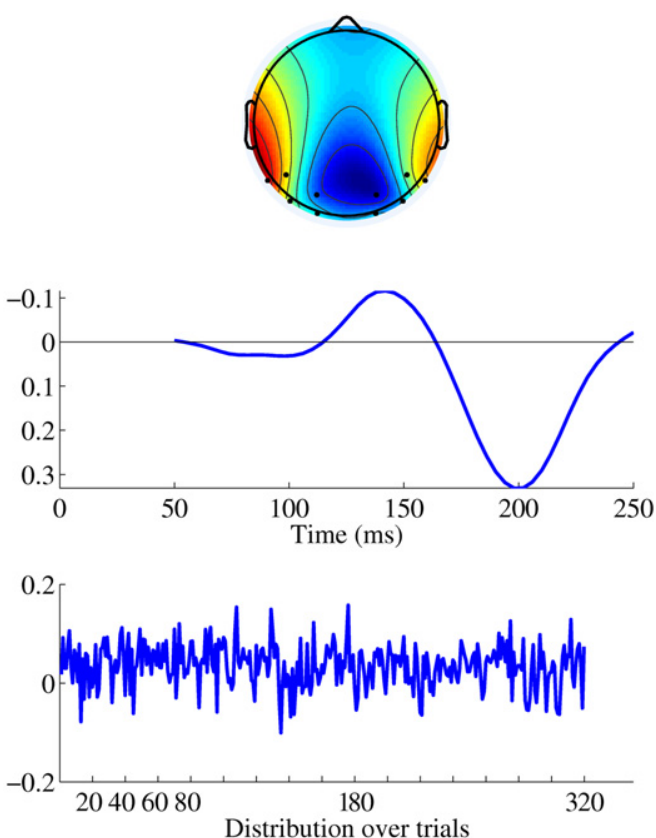
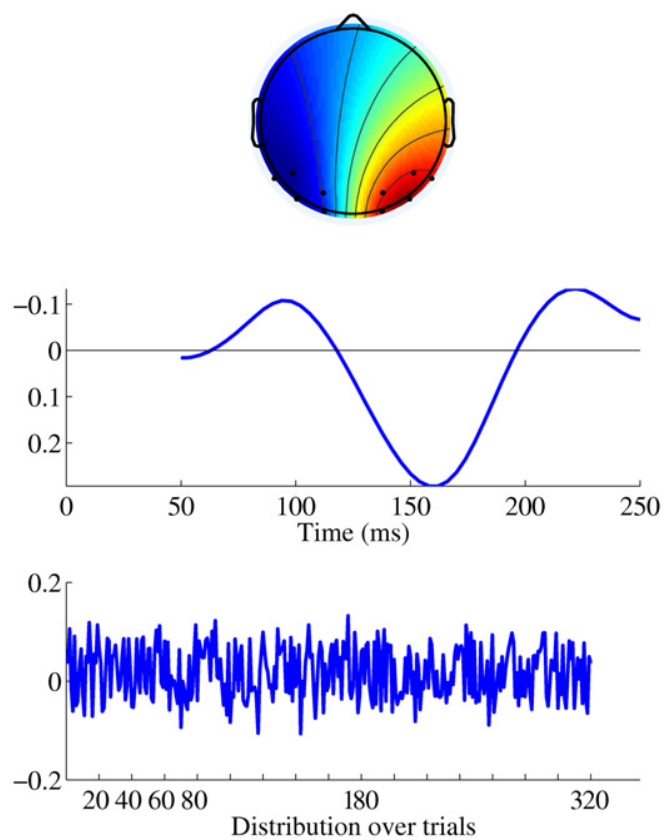

Figure 2. Illustration of a PARAFAC decomposition on Channels $\times$ Time $\times$ Trials data from one subject. Two components (orthogonal in the channel mode) have been retrieved from data from the detection task from 10 occipital channels and from a limited time frame. Upper: spatial distribution of the components (only exact on the locations of the occipital electrodes, the rest of the scalp is extrapolated); Middle: time courses; Lower: distributions over trials.

\section{Application of PARAFAC to Single Trial ERP Data}

As mentioned above, in this study ERP data were arranged in arrays with dimensions Channels $\times$ Time $\times$ Trials. More specifically, trials from all peripheral stimuli (upper left, upper right, down left, and down right) were taken together in the threedimensional data array. Applying PARAFAC to this type of data arrays results in a number of atoms, each characterized by a certain spatial distribution, a certain time course, and a certain variation across trials. The number of atoms corresponds to the rank of the data array, but, as opposed to the two-dimensional case, this rank has to be empirically determined. In this study, the performance of the method was therefore investigated for the number of atoms varying from 1 to 10 .

Furthermore, since the aim of applying PARAFAC in this study was to validate its feasibility in distinguishing between different task-related conditions, it was verified whether including prior knowledge about the expected task-related differences improved the results. To this end, reducing both the number of channels and the included time range were investigated. More specifically, to distinguish between left and right stimuli, five parietal-occipital channels in each hemisphere were chosen. To distinguish between all four quadrant stimuli, the selected occipital set of channels was extended with three channels on the midline (Pz, POz, and $\mathrm{Oz}$ ). Since for both cases the results clearly improved, all further analyses were only performed on this limited set of channels. We believe that such a subset of interesting channels can also be determined for most other experimental paradigms. In the time domain, the investigated time range was the period including the P1 and N1 waves (for the left-right case) and the one around the C1, P1, and N1 waves (for the quadrant case).

Centering and rescaling. Before fitting PARAFAC to a threedimensional dataset, the data need to be preprocessed. As men- tioned in earlier studies (Field \& Graupe, 1991; Harshman \& Lundy, 1984), two ways of preprocessing the three-dimensional data can be used: centering and rescaling. Centering is accomplished by subtracting the mean in a certain mode from the data whereas rescaling is performed by dividing the data by its standard deviation in one specific mode. In the current study, centering was applied over channels (corresponding to applying an average reference to the selected subset of electrodes) while rescaling was performed in the trial mode. Also, the combination of both was investigated.

Orthogonality. A frequent problem occurring when fitting PARAFAC models is the so-called phenomenon of degeneracy (Harshman \& Lundy, 1984). This term is used for models in which the corresponding signatures of different atoms are mutually highly correlated or in which two or more atoms counteract one or more other ones. This causes components to be redundant and therefore difficult to interpret (Field \& Graupe, 1991). Field and Graupe suggested imposing orthogonality in one of the modes in order to avoid degeneracy (as also proved by Harshman \& Lundy, 1984). Since orthogonality constraints were believed to be data specific, their effect was investigated in the channel, time, and trial mode separately.

\section{Evaluation of the Results}

To test the ability of PARAFAC to capture task-specific information, we checked the possibility to distinguish between different task-related conditions based on the PARAFAC trial mode. For comparison, a similar single trial classification was also performed based on specific peak amplitudes retrieved from the raw data. In addition, the relationship between the trial-by-trial modulations of the obtained PARAFAC components and the fMRI data was investigated. 

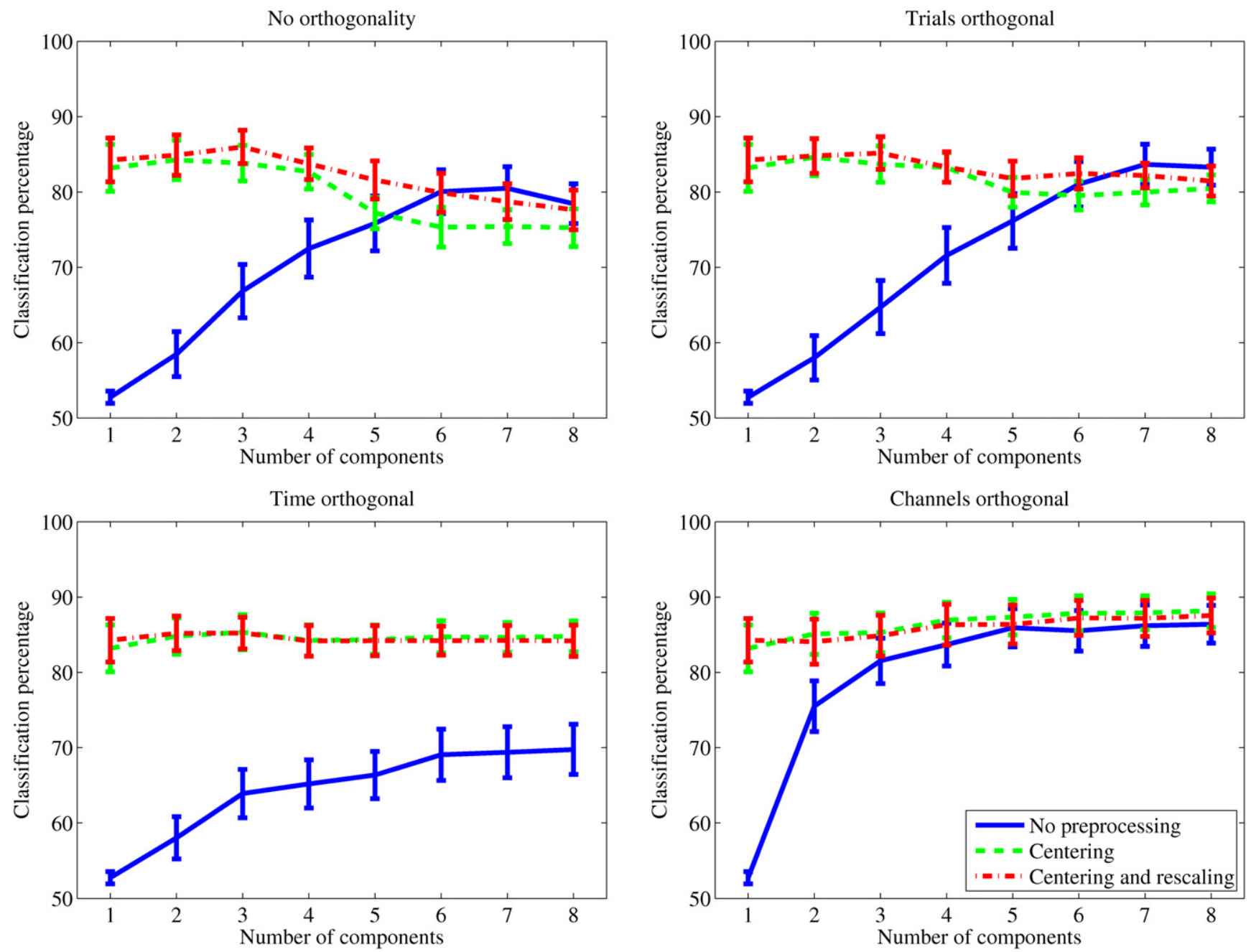

Figure 3. Effect of centering and rescaling (in different colors), orthogonality (in different subplots), and the number of extracted components (on the horizontal axis) on the PARAFAC classification accuracy for the outside data. The shown values are average (lines) and standard error values (error bars) over subjects and correspond to the analyses including temporal prior knowledge (time frame of 50-250 ms). Regardless of the number of components extracted in each case, each time only one component was used for classification.

Single trial classification based on raw data. Quantifying the stimulus-related differences in the characteristics of certain ERP components on a single trial level yields a classification between single trial ERPs belonging to different task-related conditions. The most prominent stimulus-dependent effect found in the ERP components of the detection task is the difference in latency and amplitude of the P1 and N1 peak for left versus right stimuli. For this reason, we tested two types of raw data classification, based on either the $\mathrm{P} 1$ or the $\mathrm{N} 1$ peak properties. The following approach, as originally proposed by Novitskiy et al. (2011), was pursued for each subject separately. First, the average latency of the P1 (and N1) peak was determined based on an average contralateral ERP from 10 parietal-occipital channels. Next, from every trial a P1 (or N1) difference measure was calculated by subtracting the mean from 5 right parietal-occipital channels of the average amplitude in a small window around the average P1 (N1) latency from the same mean value measured on 5 left parietal-occipital channels. Since it is known that the contralateral P1 and N1 should always precede the ipsilateral ones, the P1 difference should be positive for stimuli shown on the right and negative for stimuli shown on the left visual field, and the N1 difference should be exactly the opposite in polarity. Further, a linear discriminant analysis (LDA) was used for classifying these difference values in two groups. For this purpose, half of the trials that remained after thresholding $(50$ or $100 \mu \mathrm{V})$ were used for training and the other half for testing. The resulting classification accuracy was averaged over 1,000 randomizations.

In addition to left-right differences, our detection task data also show ERP differences for upper versus lower stimuli. Therefore, it was also tested whether it is possible to separate all four quadrant stimuli with a combination of two features. Apart from the P1 or N1 left-right difference measure, a single trial estimation of the amplitude of the $\mathrm{C} 1$ peak on the POz lead was also used. Since the P1 (and N1) differences should show opposite polarities for left versus right stimuli and the $\mathrm{C} 1$ peak should show opposite polarities for upper versus down stimuli, this combination seemed a correct criterion for classifying the raw single trials into the four quadrants. For the classification itself, again LDA was used on the 1,000 randomized training-test sets. 


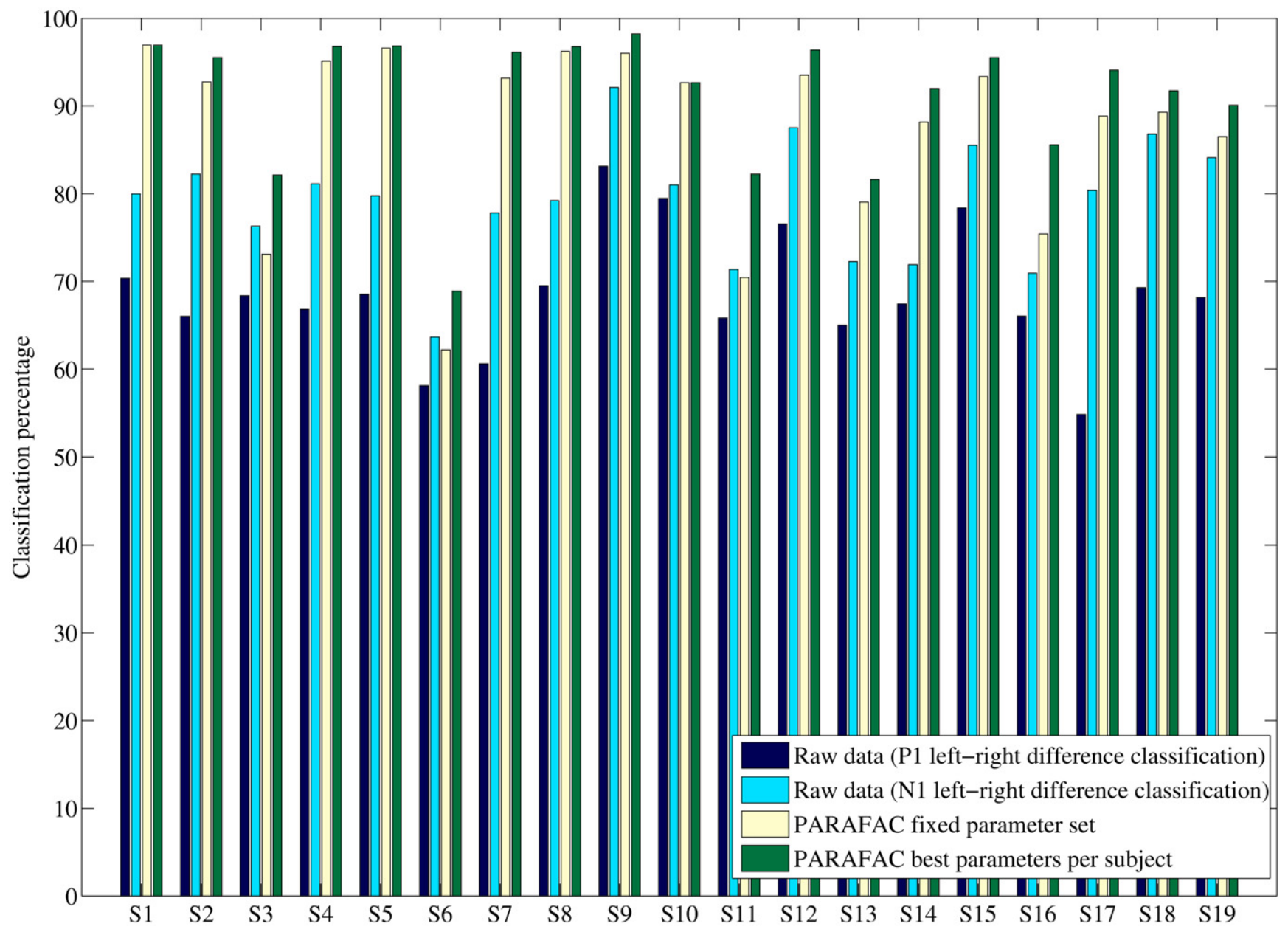

Figure 4. Left-right classification accuracy values for all subjects (indicated with letter S) with both PARAFAC and raw data classification (based on either the P1 or N1 peak) on the outside data. For PARAFAC, not only the best individual results are shown, but also results obtained by using one fixed parameter-preprocessing set for all subjects.

Single trial classification based on PARAFAC. A similar single trial classification was now aimed for with PARAFAC. In line with the classification based on the raw data, one feature was used for distinguishing between left and right stimuli, and two features were included in the differentiation between all four quadrant stimuli. The features of interest were the PARAFAC trial modes.

For the left-right difference, the trial mode of each component was fed into the LDA and, with the same repeated randomization as for the raw data, classified into two groups. This resulted in a classification accuracy for every component and for every number of extracted components and selected preprocessing steps. Afterwards, the best component and parameter-preprocessing combinations were selected.

For the quadrant-specific case, the best pair of trial modes was selected beforehand. To this end, all trial signatures were correlated with a vector based on the true left-right separation and a vector based on the true upper-down difference. The two trial modes showing the highest correlation for either one of them were fed together in the LDA and classified into four groups. The accuracy was evaluated in the same way as explained above for the left-right case.

Relation with fMRI data. As mentioned in the introduction, taskrelated information extracted on a single trial level can be used in the analysis of simultaneously acquired fMRI data. Therefore, we investigated whether the trial-by-trial fluctuations obtained with PARAFAC provided additional information for the analysis of the fMRI. However, since the ERP data were thresholded to remove bad quality trials, not all trials that were originally acquired from every subject were included in the eventual PARAFAC analysis. Having the ERP information from all originally acquired trials is nevertheless essential for connecting these to their corresponding fMRI activation.

To address this problem, we used an extrapolation of the obtained decomposition from a limited number of trials to all the trials originally acquired. As such, an estimation $C_{\text {new }}$ was obtained, representing the full trial signatures based on the channel and time signatures from the PARAFAC decomposition on a limited number of trials.

With these trial signatures, the effect of including amplitude information from the PARAFAC trial mode in the analysis of the fMRI data was investigated. The approach followed will be illustrated here on data from five subjects showing good to very good classification accuracy ( $>78 \%$ ) for distinguishing between left and right stimuli. The classification was performed here based on a K-means clustering approach (preferred here over LDA to avoid the necessity of a separate training set), clustering the trial mode of the extracted PARAFAC components into two groups. The classi- 


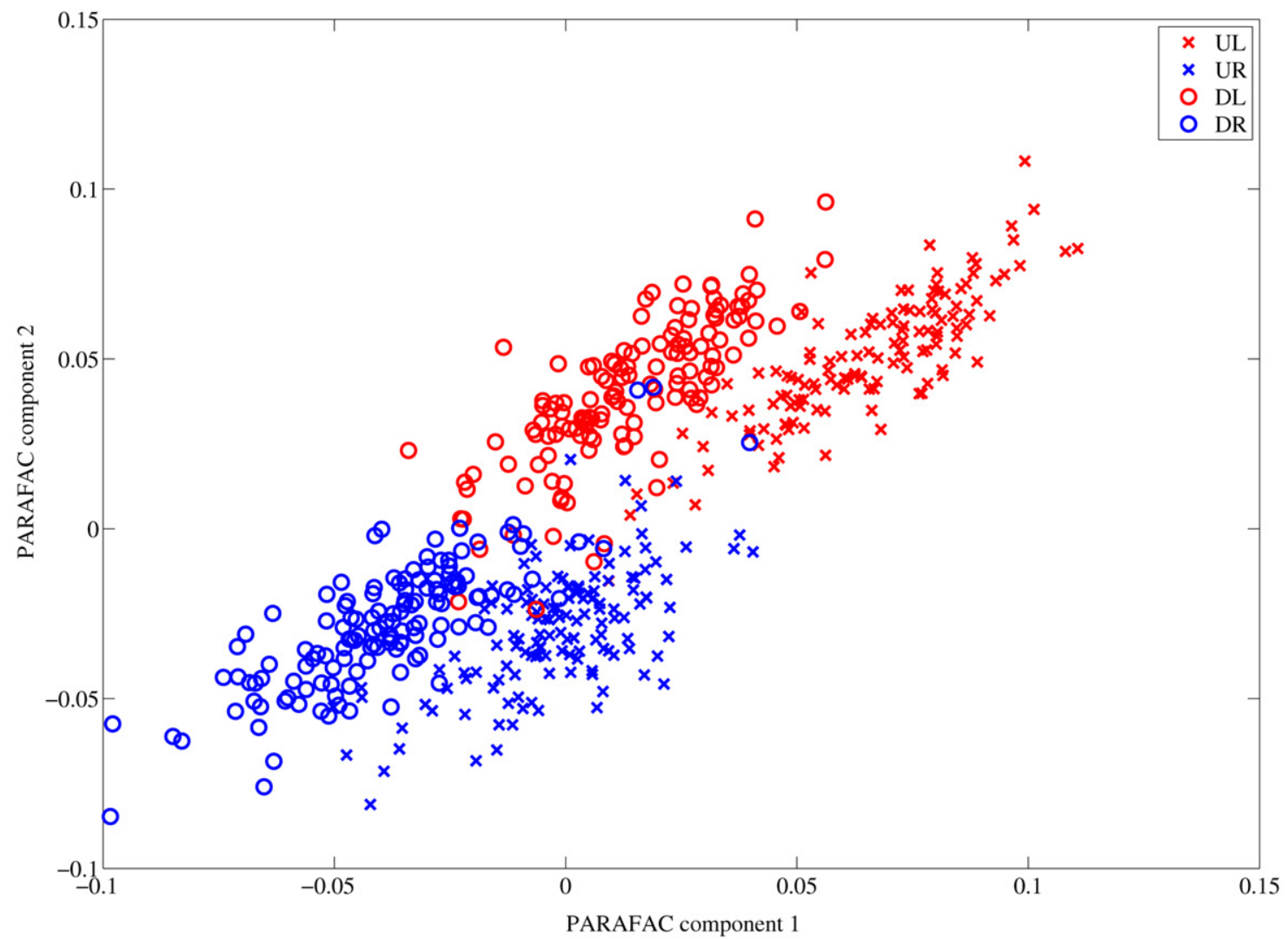

Figure 5. Illustration of the combination of two PARAFAC components for distinguishing between the four quadrant stimuli in the detection task. The results are shown for subject 5 with centered data, orthogonality imposed in the channel mode, and 10 components. The axes correspond to the trial modes of the two PARAFAC components while the colors and shapes of the marks denote the true stimulus type (UL $=$ upper left; $\mathrm{UR}=$ upper right; $\mathrm{DL}=\mathrm{down}$ left; $\mathrm{DR}=$ down right $)$.

fication accuracy from clustering in four quadrants based on inside data (see Results section) did not seem high enough for analyzing fMRI data.

Based on the above clusters, we wanted to verify whether single trial amplitude information from PARAFAC improved the model for the fMRI data. This was done by using one standard regressor based on task triggers and constructing two other regressors from the PARAFAC trial mode. For these latter two, regressors were created corresponding to each of the stimuli, thereby following the classification as explained above. However, while the first type of regressors only consisted of fixed values at the time instants of the trials (and zeros otherwise), the second type contained the actual normalized amplitude values obtained from the PARAFAC trial mode. The resulting signals were convolved with a hemodynamic response function (HRF) model and employed as regressors, each in a separate analysis, in the general linear model (GLM) explaining the fMRI data (with the SPM5 software). Afterwards, a simple $t$ test was used to summarize the results over the five subjects.

\section{Results}

\section{Grand Average ERPs of Employed Data}

To illustrate the data that have been used to obtain the results shown below, Figure 1 shows the grand average ERPs for the four peripheral stimuli (upper left, upper right, down left, and down right) for a left, a middle, and a right parietal-occipital electrode, together with the topographies at the latencies of the P1 and N1 (obtained from a window around 120 and $200 \mathrm{~ms}$, respectively). The ERP results are shown for data acquired both simultaneously and nonsimultaneously with fMRI. This figure allows visually identifying the differences between the responses to each of the peripheral stimuli, both in the ERP waveforms and in their spatial distribution.

\section{Illustration of the PARAFAC Approach}

Figure 2 shows an illustration of applying PARAFAC to a threedimensional data array from one individual subject (subject 8). The data shown here were measured outside the scanner and only the time frame around the P1 and N1 waves was included in the analysis. Two components were retrieved, of which the spatial, temporal, and trial distribution are shown.

The first component (shown on the left in Figure 2) is leftright symmetrical over channels and corresponds to the ERP information common to left and right visual field stimuli. The second component represents the difference between left and right stimuli. This can be seen in the asymmetry of the spatial distribution and can be quantified by using the trial mode for classifying between left and right stimuli, yielding a classification accuracy of $94.75 \%$. 


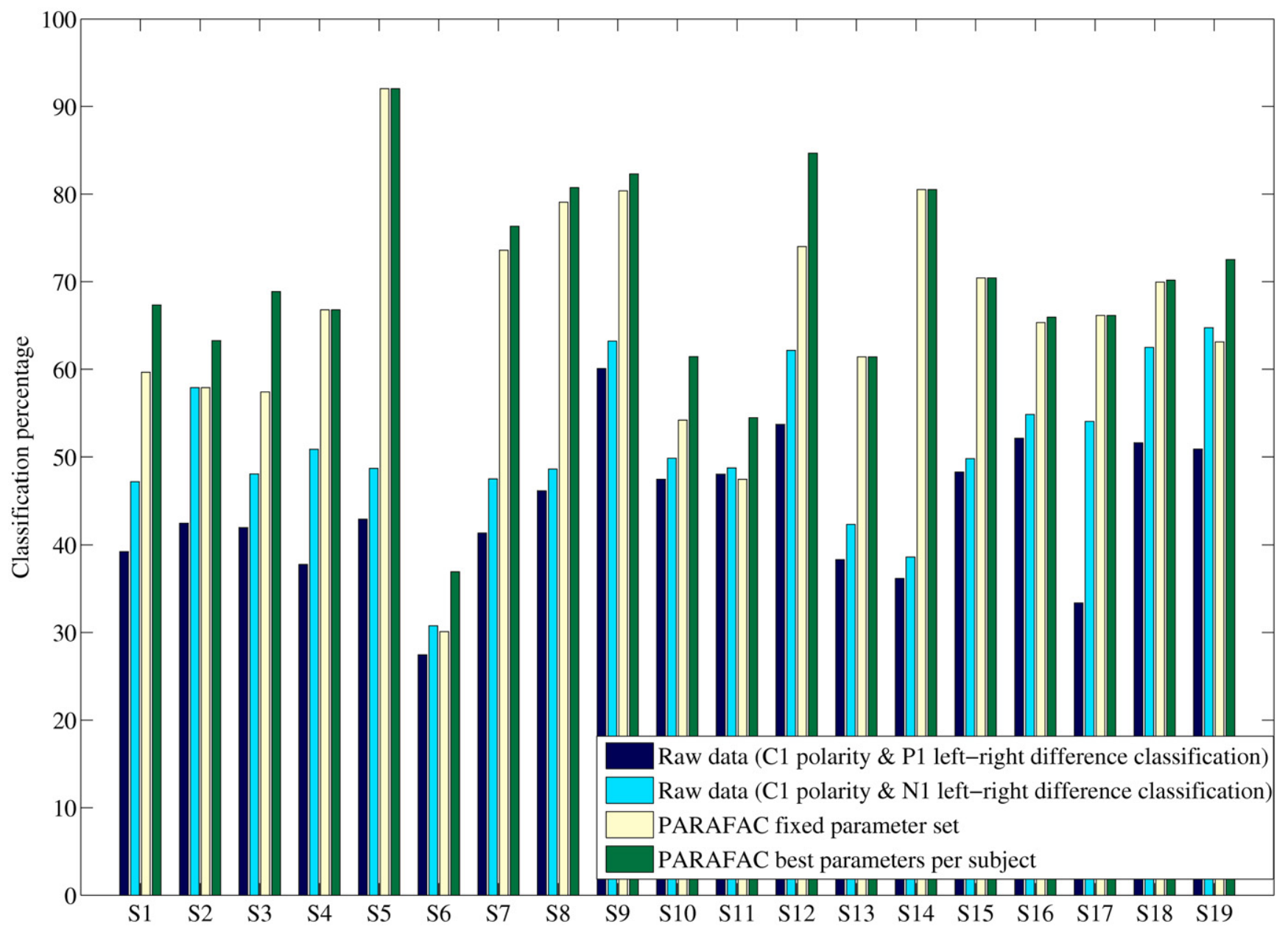

Figure 6. Classification accuracy for all subjects (indicated with letter S) with both PARAFAC and raw data classification (based on either the P1 or N1 peak) on the outside data, distinguishing the trials in the four quadrant stimuli. For PARAFAC, not only the best individual results are shown, but also results obtained by using one fixed parameter-preprocessing set for all subjects.

\section{Single Trial Left-Right Classification of Outside Data}

The influence of the number of extracted components, the choice of orthogonality, and the exact preprocessing steps on the average classification accuracy across subjects is shown in Figure 3 for the limited time frame (50-250 ms).

Without centering or rescaling, imposing orthogonality in the channel domain requires fewer components for a reasonable classification accuracy (i.e., $80 \%$ on average for three components) than orthogonality in trials or using no orthogonality at all. With centering, this dependence on the number of extracted components and orthogonality seems to almost disappear, such that the importance of careful parameter selection decreases to a great extent.

In Figure 4, the classification accuracy values from PARAFAC on the outside detection task data are compared with the ones retrieved from the raw data (based on either the $\mathrm{P} 1$ or the $\mathrm{N} 1$ peak). For PARAFAC, not only the best individual results are shown, but also results obtained by using one fixed parameter-preprocessing set for all subjects. The choices made for the fixed parameter set here are using data with only the limited time frame (50-250 ms) included, applying centering and obtaining 5 components that are orthogonal in the channel domain.

From this figure, it is clear that PARAFAC returns both a significantly higher classification accuracy compared to the
P1-based raw data classification (Wilcoxon signed rank test: $p=1.3 \cdot 10^{-4}$ for both fixed and best case) and to the N1-based raw data classification $\left(p=4.6 \cdot 10^{-4}\right.$ and $p=1.3 \cdot 10^{-4}$ for the fixed and best case, respectively).

\section{Single Trial Quadrant Classification of Outside Data}

When aiming at distinguishing between all four quadrant stimuli, two components need to be combined, one component explaining the difference between left versus right stimuli and another component related to the upper-down difference. An illustration of the combination of two PARAFAC components, revealing a clear distinction between the four stimuli, is shown in Figure 5 (subject 5). The values on the axes correspond to the respective trial signatures of two components from a specific PARAFAC decomposition, whereas the colors and shapes indicate to which stimulus these points actually belong.

Figure 6 summarizes the results of classifying between all four quadrant stimuli based on both PARAFAC and the raw data. Similar to the results of the left-right classification, here also the best results are compared with the accuracy obtained when using one specific parameter set for all subjects. For this fixed parameter set, centering was used on the data with a limited time frame 


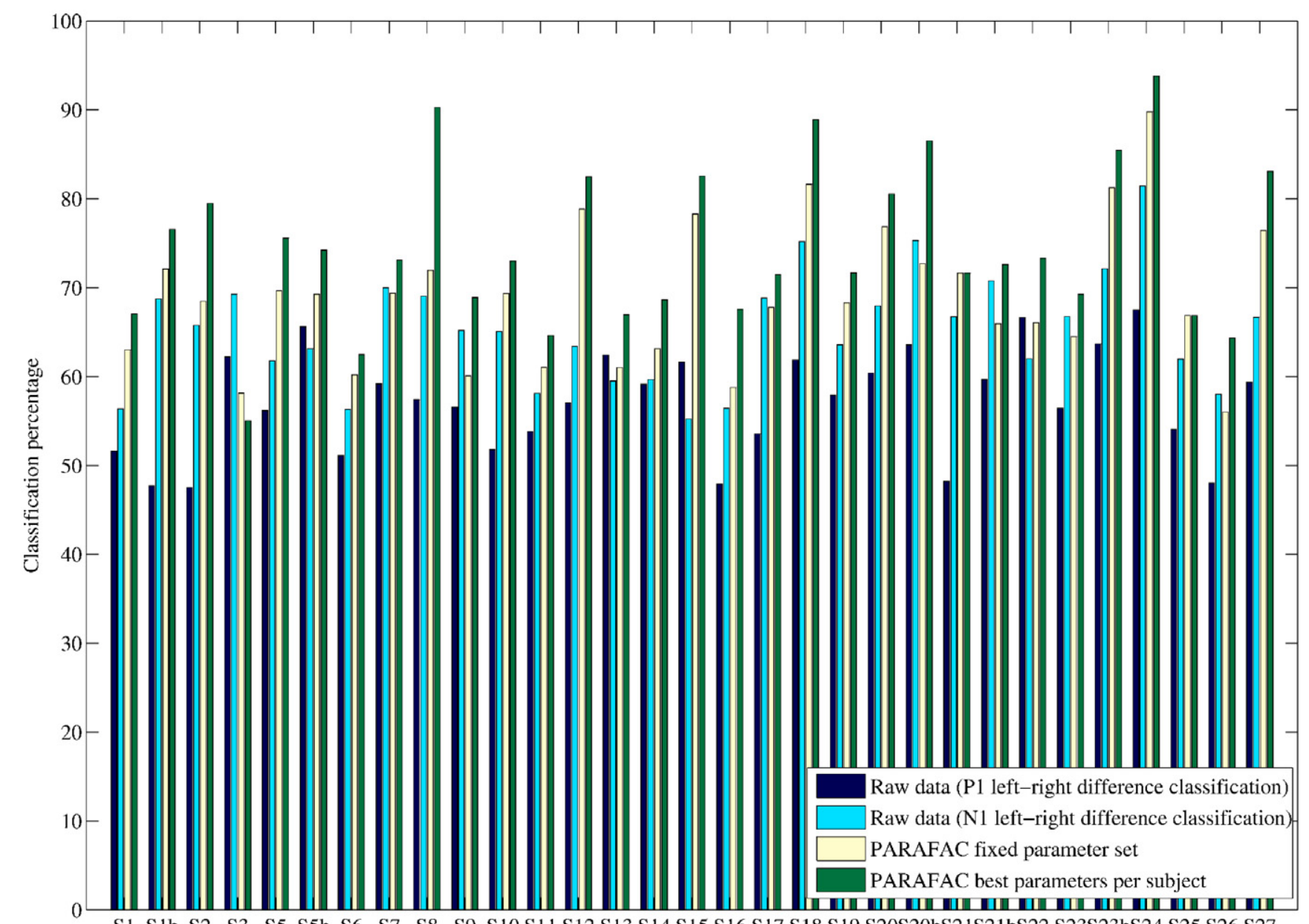

S1 S1b S2 S3 S5 S5b S6 S7 S8 S9 S10 S11 S12 S13 S14 S15 S16 S17 S18 S19 S20S20bS21S21bS22 S23S23bS24 S25 S26 S27

Figure 7. Left-right classification percentages for all subjects (indicated with letter S) with both PARAFAC and raw data classification (based on either the P1 or N1 peak) on the inside data. For PARAFAC, not only the best individual results are shown, but also results obtained by using one fixed parameter-preprocessing set for all subjects. For five of the subjects, data measured in a Siemens scanner were also included; these datasets are indicated with the letter b. Subject 4 is not included in this figure, since for this subject, only outside but no inside data were available.

(30-250 ms), after which a decomposition in 10 components with orthogonalization in the channel mode was applied.

This figure clearly shows that PARAFAC outperforms the raw data classification also when used to separate all four peripheral stimuli. This effect is confirmed statistically for both the P1- and N1-based cases and for both the best and fixed parameter set $(p$ values of Wilcoxon signed rank test: $\mathrm{P} 1$ : respectively, $1.3 \cdot 10^{-4}$ and $1.6 \cdot 10^{-4}$; N1: respectively, $1.3 \cdot 10^{-4}$ and $6.3 \cdot 10^{-4}$ ).

\section{Single Trial Left-Right Classification of Inside Data}

Due to the more severe contamination of the inside data, another, more stringent, threshold was chosen $(50 \mu \mathrm{V})$. However, as a consequence, the number of trials included in the inside PARAFAC classification is seriously reduced compared to the original number of trials and compared to the data outside the scanner. More specifically, for the 31 datasets ( 26 subjects of which 5 were measured in two different scanners), on average $67 \%$ of the trials were retained after thresholding (with a minimum of $45 \%$ ).

In Figure 7, classification accuracy values from PARAFAC on the inside data are compared with the ones retrieved on the raw data. For PARAFAC, not only the best individual results are shown, but also results obtained by using one fixed parameterpreprocessing set for all subjects (9 components, limited time frame, centering, and orthogonality in the trial mode).

The classification based on PARAFAC with the best parameters per subject is significantly better than the one based on the P1 and N1 left-right differences in the raw data $\left(p=1.6 \cdot 10^{-6}\right.$ and $p=1.5 \cdot 10^{-5}$, respectively). Although in this case it was more difficult to find one fixed parameter set, the results are also significantly better ( $p=2.8 \cdot 10^{-6}$ and $p=0.0014$, respectively).

\section{Single Trial Quadrant Classification of Inside Data}

Figure 8 summarizes the results of classifying between all four quadrant stimuli based on both PARAFAC and the raw data. Also, here, the best results are compared with the accuracy obtained when using one fixed parameter set for all subjects. In this case, the time-limited data were centered and rescaled, and 9 components, orthogonal in the trial mode, were extracted.

Although the performance of single trial quadrant classification here is obviously lower than in the case of outside data, PARAFAC is still better than the raw data classification. This effect is confirmed statistically for both the best and the fixed parameter set 


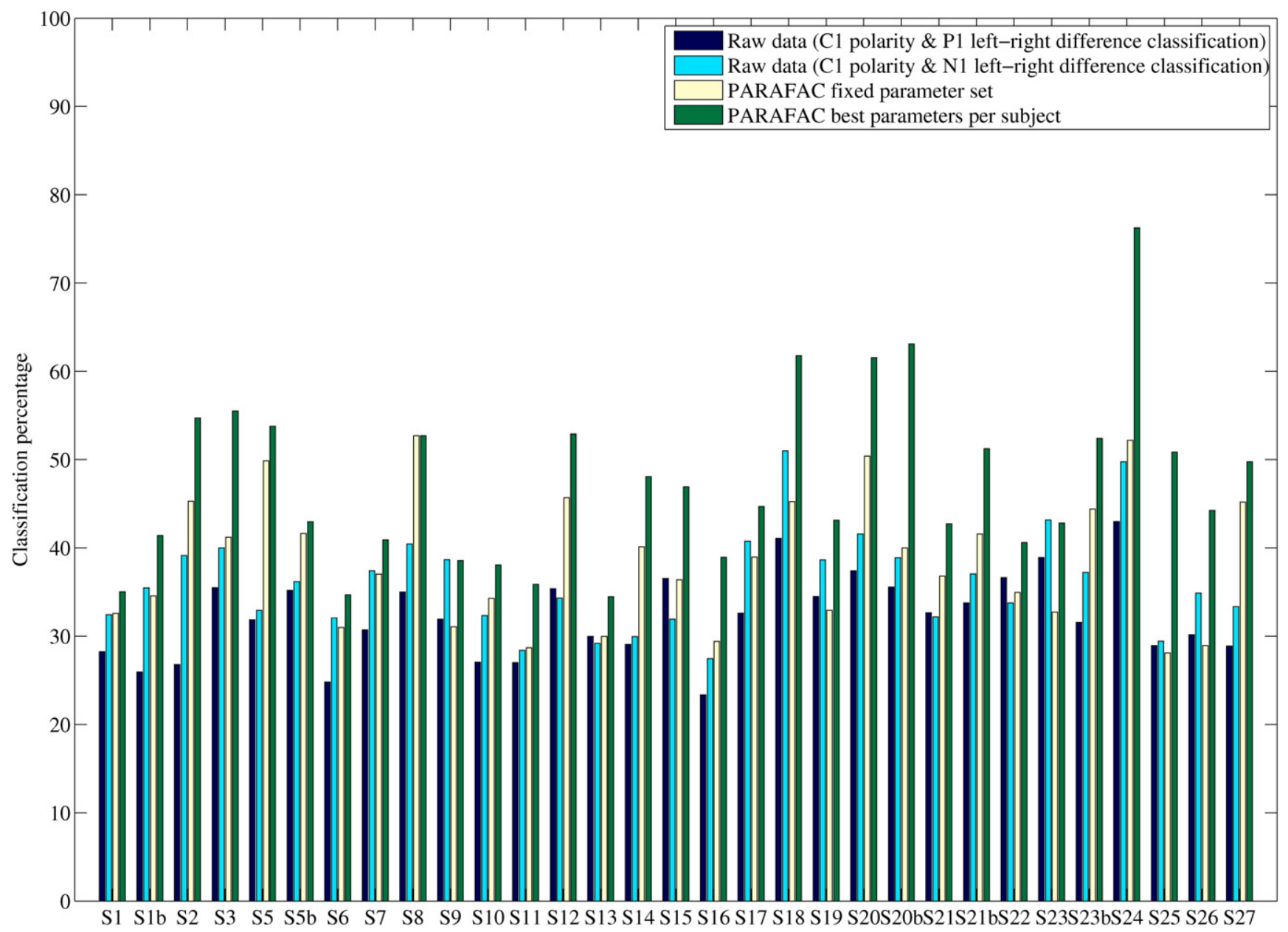

Figure 8. Classification accuracy for same subjects shown in Figure 7 (indicated with letter S) with both PARAFAC and raw data classification (based on either the P1 or N1 peak) on the inside data, distinguishing the trials in the four quadrant stimuli. For PARAFAC, not only the best individual results are shown, but also results obtained by using one fixed parameter-preprocessing set for all subjects.

( $p$ values for $\mathrm{P} 1$ of, respectively, $1.2 \cdot 10^{-6}$ and $5.9 \cdot 10^{-5}$ and for $\mathrm{N} 1$ of $1.6 \cdot 10^{-6}$ and 0.04$)$.

\section{Relation with fMRI Data}

Results shown here are based on data from five subjects. Three types of regressors were used (in separate analyses). The first one was a standard regressor based on the trigger information from the task, whereas the second one was based on the triggers according to the PARAFAC classification results. Finally, the third regressor included the amplitudes from the PARAFAC trial signatures (the $c_{t k}$ values in Equation 1) in the analysis. Activated regions obtained with the PARAFAC approach without amplitude information are summarized in Table 1 for each of the four trial types.

The obtained activations not only nicely correspond to the findings when using the original task triggers but they also match the expected regions for this type of task. This can also be seen when comparing the obtained regions with the activations shown in a study performed on the same dataset, but with a different type of analysis (Mijović et al., 2012).

Using the regressors based on the PARAFAC amplitude information revealed the same regions. However, the obtained $t$ values in these regions were lower than in the case without amplitude information. This is illustrated in Figure 9. Five small regions of interest (ROIs) were defined for each subject, based on the activation obtained using the original task triggers. These ROIs were four locations in the early visual areas, each corresponding to one specific stimulus, and a last ROI was defined in the motor area. For each location and each stimulus, the average $t$ value over all corresponding voxels and the five subjects was calculated.

Because the extraction of the different ROIs is based on the standard fMRI analysis using task triggers, it is obvious that this analysis shows the expected activations for each of the stimuli (dark blue). The analyses based upon PARAFAC (both with and without amplitudes), however, also yield these same regions. Nevertheless, there is a clear decrease in $t$ values when comparing the activation based on the amplitude regressors with the activation obtained with the trigger information from the task or the PARAFAC classification. This is confirmed statistically, showing $p$ values above .45 when comparing the triggers from PARAFAC with the task, but resulting in $p$ values between .03 and .08 and between .007 and .05 when comparing the amplitude approach with the approaches based on task and classification triggers, respectively. As such, it can be seen that using the ampli- 
Table 1. Regions Obtained by Using the Classified Trial Information as Input for the GLM Analysis in SPM

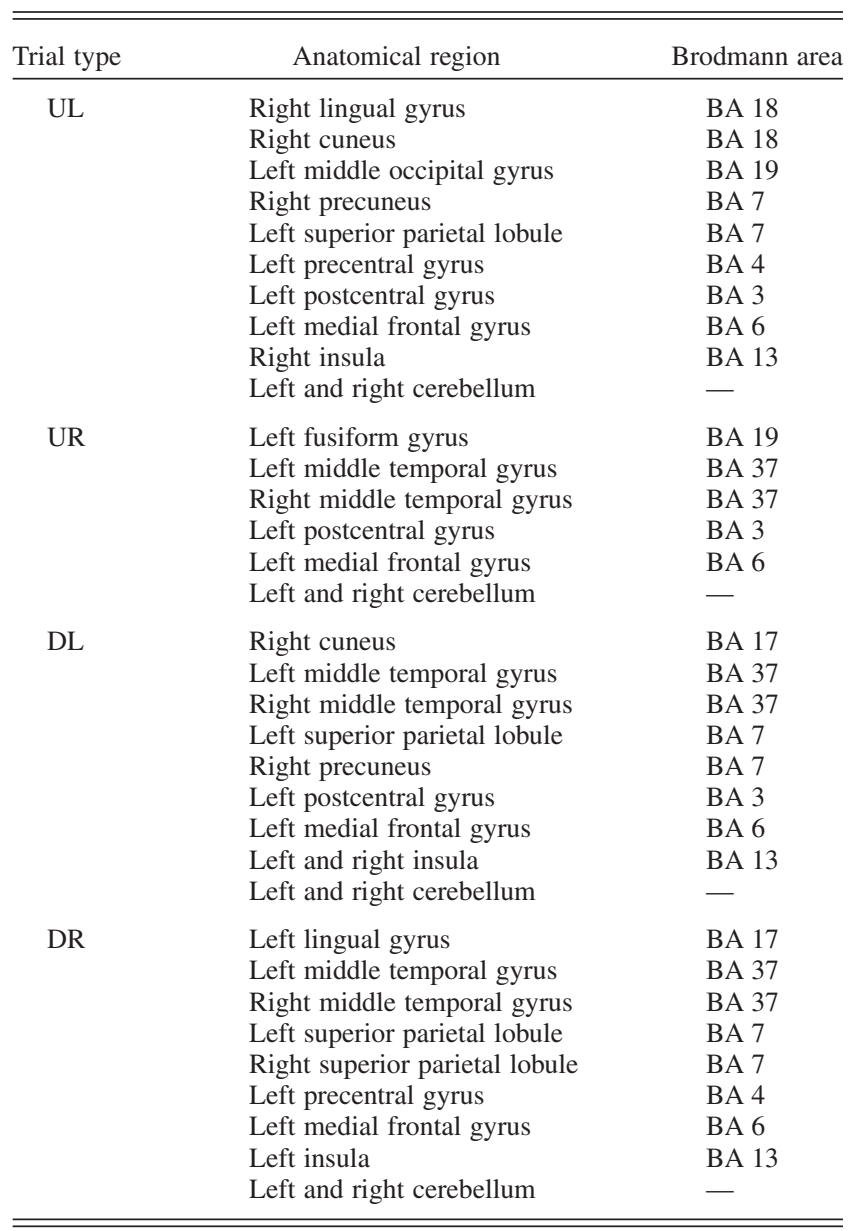

Notes. The difference between upper and lower stimuli is based on the task itself, while the left-right classification is based on the PARAFAC output. As such, regions are retrieved for upper left (UL), upper right (UR), down left (DL) and down right (DR) stimuli. For every region, both its anatomical name and its Brodmann area (BA) are given.

tudes from the trial mode explaining most of the left-right differences seems to deteriorate the fMRI results.

\section{Discussion}

In this study, we explored the ability of the higher-order decomposition method PARAFAC to extract specific task-related information from single trial ERP data. The results were validated by using the obtained components for classifying between different taskrelated conditions and by comparing these results with a similar classification based on raw data. Both data acquired in a control room and data acquired inside an MR scanner simultaneously with fMRI were used. The latter not only allowed testing the robustness of the method on data with a lower signal quality, but also enabled including the obtained single trial amplitude values (from the PARAFAC signatures in the trial mode) in the analysis of the fMRI data.

Our results indicated that PARAFAC is indeed able to capture components reflecting task-related ERP differences on a single trial basis. This was clearly shown for both the left versus right stimuli and for the four quadrant stimuli. Careful parameter tuning was, however, needed. The results were significantly better than when only raw data characteristics were used for classification, possibly suggesting an improved de-noising and a more complete and relevant feature selection achieved with PARAFAC. Also the data measured inside the MR scanner allowed this classification, although the lower signal quality influenced the performance of the approach. Since the PARAFAC trial mode was found to be a meaningful representation of task-related information, its amplitude information was also investigated in the context of EEG-fMRI integration. However, although fMRI results based on the PARAFAC classification were similar to the ones based on standard analyses, including the amplitude values seemed to reduce the strength of the obtained activations.

Using PARAFAC for classifying between different conditions on a single trial basis has certain advantages. First of all, the method is relatively fast, especially when extracting lower numbers of components (for which centering can be beneficial). More specifically, the computational time varies from less than a second to maximally 1 or $2 \mathrm{~min}$, the latter being for larger datasets and a larger number of extracted components $(4 \times$ Dual-Core AMD Opteron processor $8214,2.2 \mathrm{GHz}, 16 \mathrm{~GB}$ RAM). This is particularly fast, given the fact that more than two modes of data are included in the analyses and can be important in certain applications (e.g., brain computer interfaces, see below). Second, as opposed to the raw data classification, PARAFAC does not require detecting specific peaks or features from the ERP data. In our case, the raw data classification might still have been improved by selecting other peaks or combining a set of peaks, but the need for peak detection and selection makes it a more laborious approach. For PARAFAC, prior knowledge concerning channels and time was used, but this selection was clearly less stringent than the one needed for the raw data classification. Third, PARAFAC also allows performing group analyses by combining the datasets of several subjects in a four-way data array with dimensions Channels $\times$ Time $\times$ Trials $\times$ Subjects. Nevertheless, such group analyses require that the order of the stimuli is the same for all subjects. As stimuli were randomized in the paradigm presented here, a group analysis was not included in the current study. A major disadvantage of the PARAFAC approach is, however, the need for parameter tuning and careful preprocessing. Nevertheless, this finding does not come as a surprise, since also in order to apply PARAFAC to average ERP data, the parameters had to be optimized for each dataset (Field \& Graupe, 1991). In any case, incorporating centering and rescaling in the preprocessing of the data is very common when using PARAFAC and also clearly improved the results in this specific application (more prominent with centering than with rescaling). Concerning validation, in this study, the results were evaluated based on a classification in different stimulus types. As many paradigms consist of different stimuli, such validation procedures should be possible in other studies as well. To find the optimal parameter and preprocessing settings, training data can be used by, for example, splitting the data in several parts (as was done in the current study) or by using the datasets from a few subjects to set the parameters for the whole population. Our results with the fixed parameter set on the data acquired outside the scanner prove that the latter strategy might also be successful.

One might also ask whether the information provided by latency shifts should be taken into account by PARAFAC to model the ERP data and the condition-specific information. For this reason, Mørup, Hansen, Arnfred, Lim, and Madsen (2008) proposed the use of so-called shifted PARAFAC for ERP data. The advantage of this approach would be that, for example, small time shifts of certain components would not completely deteriorate the 
Stimulus UL

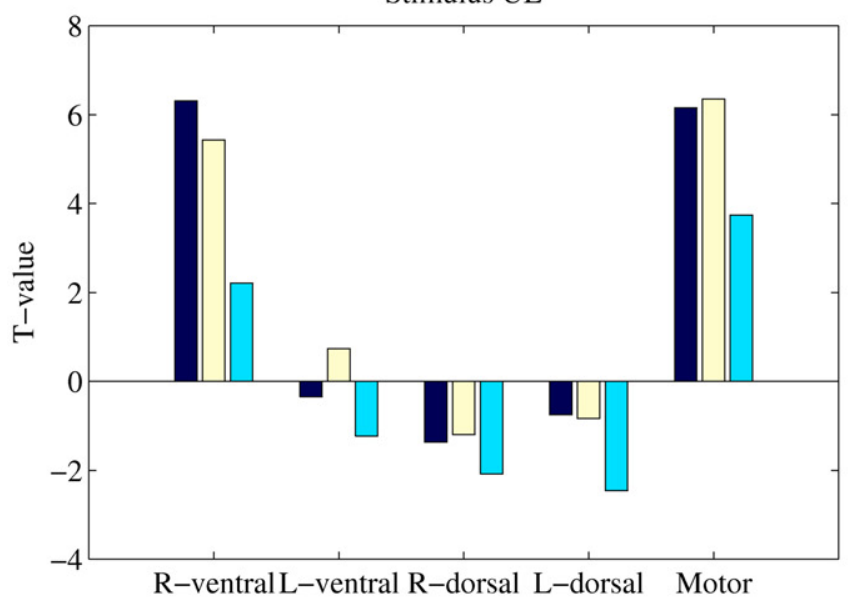

Stimulus DL

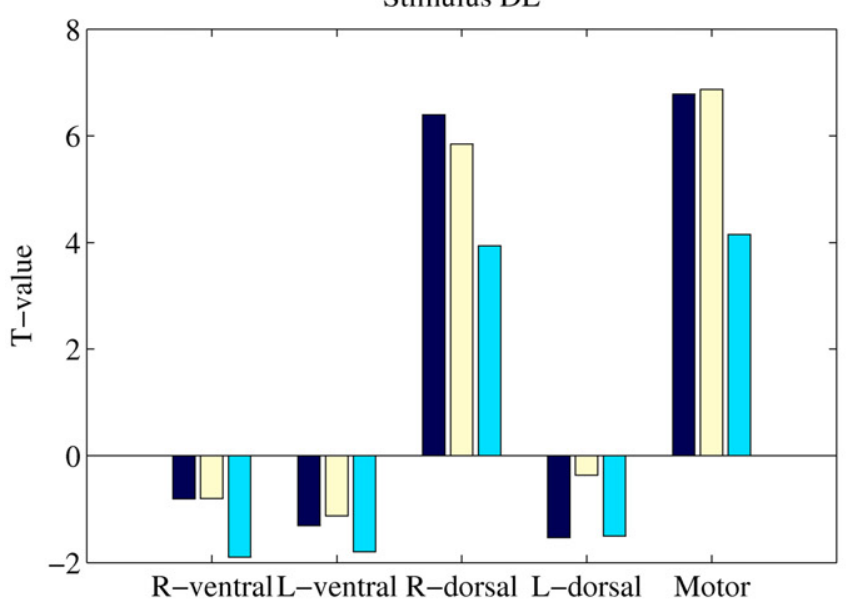

Stimulus UR

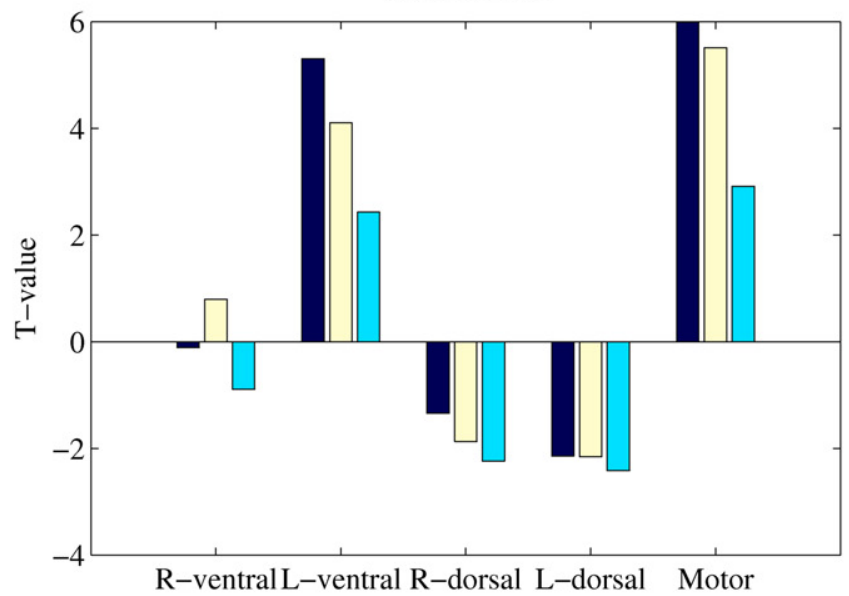

Stimulus DR

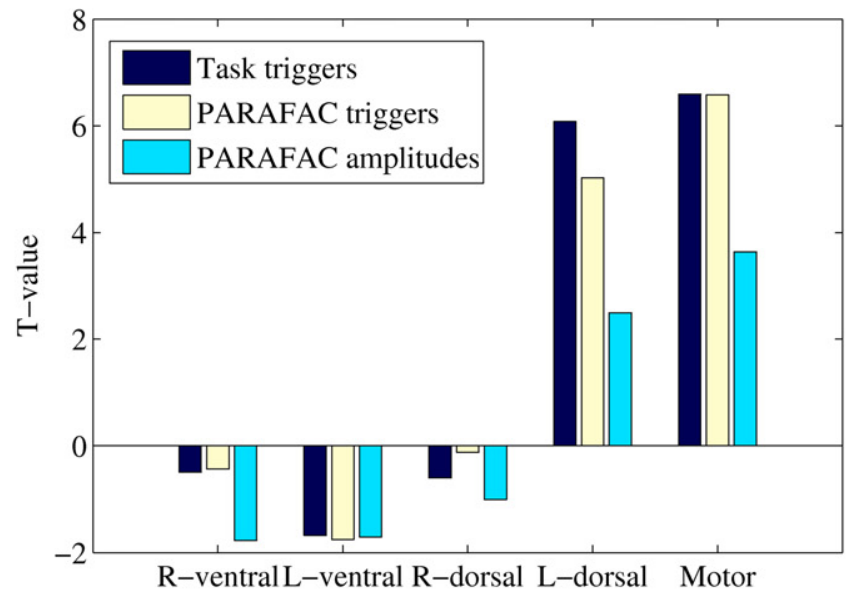

Figure 9. $T$ values in five selected ROIs (four visual regions with their position around the calcarine sulcus indicated: $\mathrm{R}=$ right; $\mathrm{L}=$ left; and a motor region) averaged over voxels and subjects and for each of the stimuli ( $\mathrm{UL}=$ upper left; $\mathrm{UR}=$ upper right; $\mathrm{DL}=$ down left; $\mathrm{DR}=$ down right). Values are shown for three different types of analyses: a standard GLM analysis based on the triggers from the task (dark blue), a similar analysis based on the left-right classification obtained with PARAFAC (light yellow), and an analysis based on regressors including PARAFAC amplitude information (light blue).

quality of the decomposition. This algorithm was, however, also tested on these data and did not yield any good results. A short simulation study (not shown here) revealed that this was probably caused by the amount of noise on the data, which the employed implementation of shifted PARAFAC (provided for MATLAB by the same authors) did not seem to be able to handle.

The obtained classification of single trial ERP data among several stimulus types makes the presented PARAFAC application also a promising approach for use in real-time brain computer interface (BCI) applications. These BCIs are systems that use brain signals for the control of one or several external devices. The use of PARAFAC in BCIs is, however, not completely new. A number of studies investigated the potential of PARAFAC for distinguishing trial types, especially in the field of motor imagery tasks (Cichocki et al., 2008; Lee, Kim, Cichocki, \& Choi, 2007) and also visually evoked potentials (Li, Zhang, \& Zhao, 2008). Nevertheless, to our knowledge, these studies work on a wavelet-transformed version of the acquired ERP data. In the current application, the shape (both amplitudes and latencies) of the ERP waveform is essential for the distinction between the different stimulus types. To capture this information in the decomposition, it is necessary to use the raw single trial data in the analysis. For this reason, we believe that the application of PARAFAC in Channels $\times$ Time $\times$ Trials dimensions, as presented here, might be a valuable alternative in specific BCI applications. We do not claim, though, that the PARAFAC classification outperforms all existing methods in the field of single trial classification. For that, we should compare them in an extensive validation study, which was not the objective of the current work.

According to Bland, Mushtaq, and Smith (2011), an essential step to study the relationship between EEG and fMRI single trial variation is to be able to distinguish between functionally significant trial-to-trial variability and variability that merely accounts for noise. Our PARAFAC approach yielded meaningful components that were related to task-specific conditions, significantly better than similar measures on raw data. For this reason, we wanted to compare the fMRI analysis based on the PARAFAC trial signatures with the standard approach of using the trigger information from the task. The results, however, did not confirm a relation between the PARAFAC trial mode and the fMRI blood oxygen leveldependent (BOLD) signal. This opens the question whether the amplitude variation across trials as captured in PARAFAC is representative for the amplitude variability across evoked responses 
and, more generally, if single trial variability measures can always be expected to correspond between ERPs and fMRI.

Our combined PARAFAC-fMRI approach starts from the assumption that the single trial variability observed in the ERPs is reflected in the fMRI data. However, it is an ongoing debate if this is always true since EEG and fMRI can, for example, differ in their sensitivity to experimental manipulations (Debener et al., 2006). Moreover, in an empirical study of Vartiainen, Liljeström, Koskinen, Renvall, and Salmelin (2011), functionally different hemodynamic and electrophysiological patterns were shown within the same task. Other studies (e.g., Becker, Reinacher, Freyer, Villringer, \& Ritter, 2011) reported relationships of the fMRI with ongoing alpha power during a cognitive task. The variability of such spontaneous brain rhythms might be so pronounced that it hides or influences the functionally relevant variability of the evoked responses. This might explain why the contribution found in a recent study of De Martino, de Borst, Valente, Goebel, and Formisano (2011) of single trial ERP modulations to the link between EEG and fMRI was very small. Further research is needed to obtain a more complete understanding of the observed phenomena.

\section{References}

Achim, A., \& Bouchard, S. (1997). Toward a dynamic topographic components model. Electroencephalography and Clinical Neurophysiology, 103, 381-385. doi: 10.1016/S0013-4694(97)96055-0

Allen, P., Josephs, O., \& Turner, R. (2000). A method for removing imaging artifact from continuous EEG recorded during functional MRI. NeuroImage, 12, 230-239. doi: 10.1006/nimg.2000.0599

Andersson, C., \& Bro, R. (2000). The N-way toolbox for MATLAB. Chemometrics \& Intelligent Laboratory Systems, 52, 1-4. doi: 10.1016/ S0169-7439(00)00071-X

Becker, R., Reinacher, M., Freyer, F., Villringer, A., \& Ritter, P. (2011). How ongoing neuronal oscillations account for variability of evoked fMRI responses. Journal of Neuroscience, 32, 11016-11027. doi: 10.1523/jneurosci.0210-11.2011

Bénar, C. G., Schön, D., Grimault, S., Nazarian, B., Burle, B., Roth, M., . . . Anton, J. L. (2007). Single trial analysis of oddball event-related potentials in simultaneous EEG-fMRI. Human Brain Mapping, 28, 602-613. doi: $10.1002 / \mathrm{hbm} .20289$

Bland, A., Mushtaq, F., \& Smith, D. (2011). Exploiting trial-to-trial variability in multimodal experiments. Frontiers in Human Neuroscience, 5, 1-3. doi: 10.3389/fnhum.2011.00080

Carroll, J., \& Chang, J. (1970). Analysis of individual differences in multidimensional scaling via an n-way generalization of "eckartyoung" decomposition. Psychometrika, 35, 283-319. doi: 10.1007/ BF02310791

Cichocki, A., Washizawa, Y., Rutkowski, T., Bakardjian, H., Phan, A.-H., Choi, S., ... Li, Y. (2008). Noninvasive BCIs: Multiway signalprocessing array decompositions. Computer, 41, 34-42. doi: 10.1109/ MC.2008.431

De Martino, F., de Borst, A., Valente, G., Goebel, R., \& Formisano, E. (2011). Predicting EEG single trial responses with simultaneous fMRI and relevance vector machine regression. NeuroImage, 56, 826-836. doi: 10.1016/j.neuroimage.2010.07.068

De Vos, M., De Lathauwer, L., \& Van Huffel, S. (2011). Spatially constrained ICA algorithms with applications in EEG processing. Signal Processing, 91, 1963-1972. doi: 10.1016/j.sigpro.2011.02.019

De Vos, M., De Lathauwer, L., Vanrumste, B., Van Huffel, S., \& Van Paesschen, W. (2007). Canonical decomposition of ictal scalp EEG and accurate source localisation: Principles and simulation study. [Special issue]. Computational Intelligence and Neuroscience, EEG/MEG, 2007, 1-10. doi: 10.1155/2007/58253

De Vos, M., Riès, S., Vanderperren, K., Vanrumste, B., Alario, F.-X., Van Huffel, S., \& Burle, B. (2010). Removal of muscle artifacts from EEG recordings of spoken language production. Neuroinformatics, 8 , 135150. doi: 10.1007/s12021-010-9071-0

De Vos, M., Vergult, A., De Lathauwer, L., De Clercq, W., Van Huffel, S., Dupont, P., . . Van Paesschen, W. (2007). Canonical decomposition of ictal scalp EEG reliably detects the seizure onset zone. NeuroImage, 37, 844-854. doi: 10.1016/j.neuroimage.2007.04.041

Debener, S., Ullsperger, M., Siegel, M., \& Engel, A. (2006). Single-trial EEG-fMRI reveals the dynamics of cognitive function. Trends in Cognitive Sciences, 10, 558-563. doi: 10.1016/j.tics.2006.09.010

Debener, S., Ullsperger, M., Siegel, M., Fiehler, K., von Cramon, D., \& Engel, A. (2005). Trial-by-trial coupling of concurrent electroencephalogram and functional magnetic resonance imaging identifies the dynamics of performance monitoring. The Journal of Neuroscience, 25, 11730-11737. doi: 10.1523/jneurosci.3286-05.2005

Deburchgraeve, W., Cherian, P., De Vos, M., Swarte, R., Blok, J., Visser, G., .. Van Huffel, S. (2009). Neonatal seizure localization using
PARAFAC decomposition. Clinical Neurophysiology, 120, 1787-1796. doi: 10.1016/j.clinph.2009.07.044

Di Russo, F., Martinez, A., Sereno, M., Pitzalis, S., \& Hillyard, S. (2002). Cortical sources of the early components of the visual evoked potential. Human Brain Mapping, 15, 95-111. doi: 10.1002/hbm.10010

Eichele, T., Calhoun, V., \& Debener, S. (2009). Mining EEG-fMRI using independent component analysis. International Journal of Psychophysiology, 73, 53-61. doi: 10.1016/j.ijpsycho.2008.12.018

Eichele, T., Specht, K., Moosmann, M., Jongsma, M., Quiroga, R., Nordby, H., \& Hugdahl, K. (2005). Assessing the spatiotemporal evolution of neuronal activation with single-trial event-related potentials and functional MRI. Proceedings of the National Academy of Sciences of the United States of America, 102, 17798-17803. doi: 10.1073/ pnas. 0505508102

Field, A., \& Graupe, D. (1991). Topographic component (parallel factor) analysis of multichannel evoked potentials: Practical issues in trilinear spatiotemporal decomposition. Brain Topography, 3, 407-423.

Harshman, R. (1970). Foundations of the CP procedure: Models and conditions for an "explanatory" multi-modal factor analysis. UCLA Working Papers in Phonetics, 16, 1-84. doi: 10.1.1.144.5652

Harshman, R., \& Lundy, M. (1984). Data preprocessing and the extended PARAFAC model. In H. Law, C. Snyder, Jr., J. Hattie, \& R. McDonald (Eds.), Research methods for multimode data analysis (pp. 216-284). New York, NY: Praeger.

Joyce, C., Gorodnitsky, I., \& Kutas, M. (2004). Automatic removal of eye movement and blink artifacts from EEG data using blind component separation. Psychophysiology, 41, 313-325. doi: 10.1111/j.14698986.2003.00141

Lee, H., Kim, Y., Cichocki, A., \& Choi, S. (2007). Nonnegative tensor factorization for continuous EEG classification. International Journal of Neural Systems, 17, 305-317. doi: 10.1.1.81.3785

Li, J., Zhang, L., \& Zhao, Q. (2008). Pattern classification of visual evoked potentials based on parallel factor analysis. In R. Wang, E. Shen, \& F. Gu (Eds.), Advances in cognitive neurodynamics ICCN 2007 (pp. 571575). Dordrecht, The Netherlands: Springer. doi: 10.1007/978-1-40208387-7_99

Makeig, S., Jung, T.-P., Bell, A., Ghahremani, D., \& Sejnowski, T. (1997). Blind separation of auditory event-related brain responses into independent components. Proceedings of the National Academy of Sciences of the United States of America, 94, 10979-10984. doi: 10.1073/ pnas.94.20.10979

Makeig, S., Westerfield, M., Jung, T.-P., Covington, J., Townsend, J., Sejnowski, T., \& Courchesne, E. (1999). Functionally independent components of the late positive event-related potential during visual spatial attention. Journal of Neuroscience, 19, 2665-2680. doi: 10.1.1.164.3923

Makeig, S., Westerfield, M., Jung, T.-P., Enghoff, S., Townsend, J., Courchesne, E., \& Sejnowski, T. J. (2002). Dynamic brain sources of visual evoked responses. Science, 295, 690-694. doi: 10.1126/ science. 1066168

Mijović, B., Vanderperren, K., Novitskiy, N., Vanrumste, B., Stiers, P., Van den Bergh, B., ... De Vos, M. (2012). The "why" and "how" of JointICA: Results from a visual detection task. NeuroImage, 60, 11711185. doi: 10.1016/j.neuroimage.2012.01.063

Miwakeichi, F., Martínez-Montes, E., Valdés-Sosa, P., Nishiyama, N., Mizuhara, H., \& Yamaguchi, Y. (2004). Decomposing EEG data into space-time-frequency components using parallel factor analysis. NeuroImage, 22, 1035-1045. doi: 10.1016/j.neuroimage.2004.03.039 
Möcks, J. (1988a). Decomposing event-related potentials: A new topographic components model. Biological Psychology, 26, 199-215. doi: 10.1016/0301-0511(88)90020-8

Möcks, J. (1988b). Topographic components model for event-related potentials and some biophysical considerations. IEEE Transactions on Biomedical Engineering, 35, 482-484. doi: 10.1109/10.2119

Mørup, M., Hansen, L., Arnfred, S., Lim, L.-H., \& Madsen, K. (2008). Shift-invariant multilinear decomposition of neuroimaging data. $\mathrm{Neu}$ roImage, 42, 1439-1450. doi: 10.1016/j.neuroimage.2008.05.062

Mørup, M., Hansen, L., Herrmann, C., Parnas, J., \& Arnfred, S. (2006). Parallel factor analysis as an exploratory tool for wavelet transformed event-related EEG. NeuroImage, 29, 938-947. doi: 10.1016/ j.neuroimage.2005.08.005

Moosmann, M., Schonfelder, V., Specht, K., Scheeringa, R., Nordby, H., \& Hugdahl, K. (2009). Realignment parameter-informed artefact correction for simultaneous EEG-fMRI recordings. NeuroImage, 45, 11441150. doi: 10.1016/j.neuroimage.2009.01.024

Niazy, R., Beckmann, C., Iannetti, G., Brady, J., \& Smith, S. (2005). Removal of FMRI environment artifacts from EEG data using optimal basis sets. NeuroImage, 28, 720-737. doi: 10.1016/j.neuroimage. 2005.06.067

Novitskiy, N., Ramautar, J., Vanderperren, K., De Vos, M., Mennes, M., Mijović, B., ... Wagemans, J. (2011). The BOLD correlates of the visual $\mathrm{P} 1$ and $\mathrm{N} 1$ in single-trial analysis of simultaneous EEG-fMRI recording during a spatial detection task. NeuroImage, 54, 824-835. doi: 10.1016/j.neuroimage.2010.09.041

Smilde, A., Bro, R., \& Geladi, P. (2004). Multi-way analysis with applications in the chemical sciences. West Sussex, UK: John Wiley \& Sons. doi: 10.1002/0470012110

Vanderperren, K., De Vos, M., Ramautar, J., Novitskiy, N., Mennes, M., Assecondi, S., ... Van Huffel, S. (2010). Removal of BCG artifacts from EEG recordings inside the MR scanner: A comparison of methodological and validation-related aspects. NeuroImage, 50, 920-934. doi: 10.1016/j.neuroimage.2010.01.010

Vartiainen, J., Liljeström, M., Koskinen, M., Renvall, H., \& Salmelin, R. (2011). Functional magnetic resonance imaging blood oxygenation level-dependent signal and magnetoencephalography evoked responses yield different neural functionality in reading. The Journal of Neuroscience, 31, 1048-1058. doi: 10.1523/jneurosci.3113-10.2011

Wang, K., Begleiter, H., \& Porjesz, B. (2000). Trilinear modeling of eventrelated potentials. Brain Topography, 12, 263-271. doi: 10.1023/ A: 1023455404934

Wesensten, N. J., Badia, P., \& Harsh, J. (1990). Time of day, repeated testing, and interblock interval effects on P300 amplitude. Physiology \& Behavior, 47, 653-658. doi: 10.1016/0031-9384(90)90073-D

(ReceIved November 7, 2011; ACCEPTED May 12, 2012) 